\title{
Peregrine Rogue Waves Generated by the Interaction and Degeneration of Soliton-Like Solutions: Derivative Nonlinear Schrödinger Equation
}

\author{
Haoqi Zhou' ${ }^{1}$, Shuwei Xu1 ${ }^{\text {1* }}$, Maohua $\mathrm{Li}^{2}$ \\ ${ }^{1}$ College of Mathematics Physics and Information Engineering, Jiaxing University, Jiaxing, China \\ ${ }^{2}$ School of Mathematics and Statistics, Ningbo University, Ningbo, China \\ Email: *xshuwei666@126.com
}

How to cite this paper: Zhou, H.Q., Xu, S.W. and Li, M.H. (2020) Peregrine Rogue Waves Generated by the Interaction and Degeneration of Soliton-Like Solutions: Derivative Nonlinear Schrödinger Equation. Journal of Applied Mathematics and Physics, 8, 2824-2835.

https://doi.org/10.4236/jamp.2020.812208

Received: November 10, 2020

Accepted: December 12, 2020

Published: December 15, 2020

Copyright $\odot 2020$ by author(s) and Scientific Research Publishing Inc. This work is licensed under the Creative Commons Attribution International License (CC BY 4.0).

http://creativecommons.org/licenses/by/4.0/

(c) (i) Open Access

\begin{abstract}
We study the Peregrine rogue waves within the framework of Derivative Nonlinear Schrödinger equation, which is used to describe the propagation of Alfven waves in plasma physics and sub-picosecond or femtosecond pulses in nonlinear optics. The interaction and degeneration of two soliton-like solutions and its relations for the breather solution have been analyzed. The Peregrine rogue waves have been considered from the two kinds of formation processes: it can be generated through the limitation of the infinitely large period of the breather solutions, and it can be interpreted as the soliton-like solutions with different polarities. As a special example, a special Peregrine rogue wave is generated by a breather solution and phase solution, which is given by the trivial seed (zero solution).
\end{abstract}

\section{Keywords}

Derivative Nonlinear Schrödinger Equation, Breather Solution, Phase Solution, Soliton-Like Solutions, Peregrine Rogue Waves, Darboux Transformation

\section{Introduction}

In the past years, rogue waves, commonly defined as gigantic waves appearing from nowhere and disappearing without trace, have attracted a lot of attention in deep ocean waves [1], optical fibers [2] [3], and water tanks [4] [5]. The appearance of rogue waves can be related to the modulation instability of the wave background [6] [7]. The focusing nonlinear Schrödinger (NLS) equation, 


$$
i \psi_{t}+\frac{1}{2} \psi_{x x}+\psi^{2} \psi^{*}=0
$$

is commonly considered as a classic model to describe rogue waves. As it has a rich of many exact solutions due to its integrability, the Peregrine soliton [8],

$$
\psi=\left(1-4 \frac{1+2 i t}{1+4 t^{2}+4 x^{2}}\right) \mathrm{e}^{i t},
$$

possesses a high amplitude and two hollows and is the prototype of the rogue waves, which "appear from nowhere and disappear without a trace" [9]. The Peregrine soliton is usually be obtained from the breather solutions, such as the Kuznetsov-Ma breather [10] [11] and the Akhmediev breather [12], of the NLS equation through the limit of the infinitely large period of the breather soliton solutions.

Considering the generalization of the type of NLS equation, the study of rogue waves in the Derivative Nonlinear Schrödinger(DNLS) equation has also caused a lot of research [13] [14] [15] [16] [17]. The DNLS equation

$$
i q_{t}-q_{x x}+i\left(q^{2} q^{*}\right)_{x}=0
$$

is originated from nonlinear optics and plasma physics. Here “*” denotes the complex conjugation, and subscript of $x$ (or $t$ ) denotes the partial derivative with respect to $x$ (or $t$ ). In nonlinear optics, the DNLS equation is used to describe the propagation of sub-picosecond or femtosecond pulses in optical fibers [18] [19] [20]. In plasma physics, the DNLS equation governs the evolution of small but finite amplitude Alfvén waves that propagate quasi-parallel to the magnetic field [21] [22]. Kaup and Newell [23] firstly obtained the one-soliton solutions under the vanishing boundary conditions by the inverse scattering transform. The $\mathrm{N}$-soliton formula [24] for the DNLS equation under vanishing boundary condition was expressed by determinants. Some special soliton solutions [25] of the DNLS equation under nonvanishing boundary conditions were obtained, and the algebraic soliton was also given. The two-soliton solution of the DNLS equation under vanishing boundary conditions and nonvanishing boundary conditions is introduced as the so-called "paired soliton" [26] [27], which is now regarded as one kind of breather solution. By introducing an affine parameter, Chen and Lam [28] revised the inverse scattering transform for the DNLS equation under nonvanishing boundary conditions, and then got the single breather solution, which can be reduced to the dark soliton and the bright soliton. The rogue waves [13] [14] [15] [16] [17] can be constructed from the degeneration of breather solutions, soliton solutions and phase solutions through the Darboux transformation (DT) [29] [30], which is a very powerful method in the study of a plethora of integrable nonlinear systems [31] [32] [33] [34]. The breather solutions are characterizing solitons which propagate on an unstable finite background. The Peregrine rogue waves are generated through the limitation of the infinitely large period of the breather solutions. The multiple soliton solutions and phase solutions collisions have played a very important role in Peregrine 
rogue waves formation. Collisions of many soliton-like structures can cause such exciting rogue wave phenomenon [35] [36]. In addition, the interest in two soliton solution stems not only from soliton molecules [37] [38] [39] in modern nonlinear optics, but also from its application, such as the formation of the structure of soliton turbulence [40] in integrable systems. Similar soliton breathing behavior is not only limited to optical systems [41], but also can be found in nonlinear fluid waves [42]. Based on previous researches [35]-[41], it is very important to study the interaction of two solitons and their related structures. In this work, we provide the interaction and degeneration of two soliton-like solutions and its relations for the breather solutions. At the same time, according to the specific reasons for the formation of Peregrine rogue waves, some classifications are given.

The structure of this paper is as follows. In Section 2, we give the analytical form of the Peregrine rogue waves by DT from a periodic solution of the DNLS equation. Based on the explicit expression and their formation process, we can get the relations between breather solutions, phase solutions, soliton solutions and Peregrine rogue waves. In Section 3, The interaction and the degeneration mechanism of two soliton-like solutions and their key properties such as its relations for the breather solution are discussed. In the limitation $\lambda_{1} \rightarrow \lambda_{c_{1}}$ and $\lambda_{2} \rightarrow \lambda_{c_{2}}\left(\lambda_{c_{1}}=\frac{1}{2}\left(\sqrt{c^{2}-2 a}-c\right) i, \quad \lambda_{c_{2}}=\frac{1}{2}\left(-\sqrt{c^{2}-2 a}-c\right) i\right)$, the two soliton-like solutions gradually degenerate into the Peregrine rogue waves under the condition $\frac{3}{8} c^{2}<a \leq \frac{1}{2} c^{2}$. Finally, we summarize our main results in Section 4 .

\section{Analytical Form of Peregrine Rogue Waves}

The analytical form of Peregrine rogue waves $q_{r}$ of the DNLS equation is

$$
\begin{aligned}
& q_{r}=\frac{c\left(-R 1-4+8 i c^{2} a t+i R 2\right)(R 1-i R 2)}{(R 1+i R 2)^{2}} \exp \left(i a\left(a t+x-c^{2} t\right)\right), \\
& R 1=-8 c^{2} a^{3} t^{2}+12 c^{4} a^{2} t^{2}-8 c^{2} a^{2} t x-6 c^{6} a t^{2}-2 c^{2} a x^{2}+8 c^{4} a t x-1, \\
& R 2=4 c^{2} a t+2 c^{2} x-6 c^{4} t .
\end{aligned}
$$

which includes both quasi-rational bright-dark solitons and Peregrine rogue waves [15]. After calculation and analysis, the Peregrine rogue waves can be expressed in the above form under the condition $\frac{3}{8} c^{2}<a$. The maximum amplitude of $\left|q_{r}\right|^{2}$ occurs at $(x=0, t=0)$ and is equal to $9 c^{2}$, and the minimum amplitude of $\left|q_{r}\right|^{2}$ occurs at $\left(x=-\frac{3\left(-c^{2}+2 a\right)}{a c} \sqrt{\frac{3}{32 a-12 c^{2}}}\right.$,

$\left.t=\frac{1}{a c} \sqrt{\frac{3}{32 a-12 c^{2}}}\right)$ and $\left(x=\frac{3\left(-c^{2}+2 a\right)}{a c} \sqrt{\frac{3}{32 a-12 c^{2}}}, \quad t=-\frac{1}{a c} \sqrt{\frac{3}{32 a-12 c^{2}}}\right)$, and is equal to 0 . Obviously, this quasi-rational solution is a Peregrine rogue wave. In Figure 1, we give the dynamic evolution diagram of Peregrine rogue waves under two different excitation mechanisms (see Figure 1(a) and Figure $1(\mathrm{c})$ ), and give the special Peregrine rogue waves (see Figure $1(\mathrm{~b})$ ) which can be 


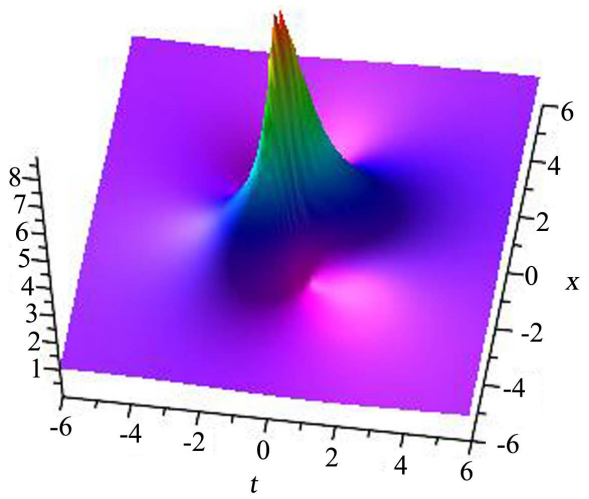

(a)

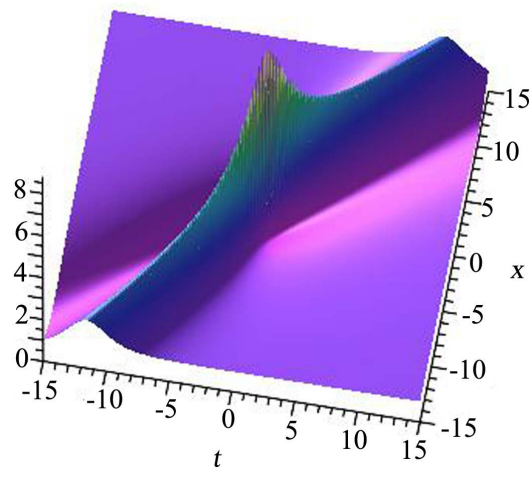

(b)

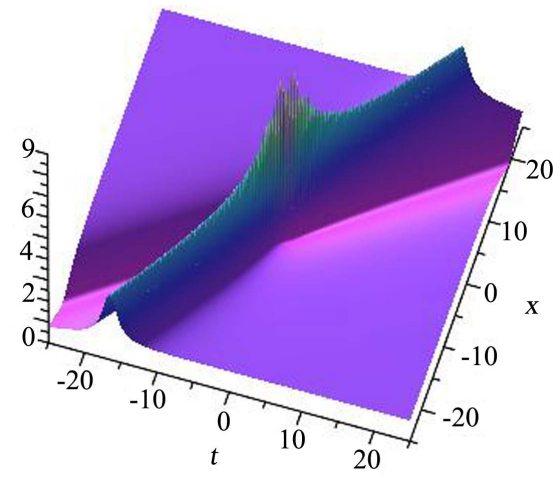

(c)

Figure 1. (Color online) The density plot of the rogue solution $\left|q_{r}\right|^{2}$ with the parameters $c=1$, (a) $a=1$, (b) $a=\frac{1}{2}$, (c) $a=\frac{7}{16}$. The Peregrine rogue waves can be formed by the breather solutions, soliton-like solutions and phase solutions under the different parameters $a$.

formed by two kinds of excitation mechanism. The specific formation mechanism is described in more detail by the collisions of two soliton-like structures in the next part.

\section{The Interaction and Degeneration of Two Soliton-Like Solutions}

The DNLS equation [23] can be given by the integrability condition $U_{t}-V_{x}+[U, V]=0$ of the following Kaup-Newell (KN) Lax pair with the reduction condition $r=-q^{*}$. The Lax pairs corresponding to the DNLS equation can be given as follows:

$$
\begin{gathered}
\partial_{x} \psi=\left(J \lambda^{2}+Q \lambda\right) \psi=U \psi, \\
\partial_{t} \psi=\left(2 J \lambda^{4}+V_{3} \lambda^{3}+V_{2} \lambda^{2}+V_{1} \lambda\right) \psi=V \psi,
\end{gathered}
$$

with

$$
\psi=\left(\begin{array}{l}
\phi \\
\varphi
\end{array}\right), \quad J=\left(\begin{array}{cc}
i & 0 \\
0 & -i
\end{array}\right), \quad Q=\left(\begin{array}{ll}
0 & q \\
r & 0
\end{array}\right)
$$




$$
V_{3}=2 Q, \quad V_{2}=J q r, \quad V_{1}=\left(\begin{array}{cc}
0 & -i q_{x}+q^{2} r \\
i r_{x}+r^{2} q & 0
\end{array}\right) .
$$

Here $\lambda$, an arbitrary complex number, is called the eigenvalue (or the spectral parameter), and $\psi$ is called the eigenfunction associated with the eigenvalue $\lambda$ of the KN Lax pair.

Next we give the general forms of the $N$-order soliton solutions [13] by using the determinant representation of the $N$-fold DT of the DNLS equation:

$$
q^{[n]}=\frac{\Omega_{11}^{2}}{\Omega_{21}^{2}} q+2 i \frac{\Omega_{11} \Omega_{12}}{\Omega_{21}^{2}} .
$$

Here, 1) for $n=2 k$,

$$
\begin{gathered}
\Omega_{11}=\left|\begin{array}{cccccc}
\lambda_{1}^{n-1} \varphi_{1} & \lambda_{1}^{n-2} \phi_{1} & \lambda_{1}^{n-3} \varphi_{1} & \cdots & \lambda_{1} \varphi_{1} & \phi_{1} \\
\lambda_{2}^{n-1} \varphi_{2} & \lambda_{2}^{n-2} \phi_{2} & \lambda_{2}^{n-3} \varphi_{2} & \cdots & \lambda_{2} \varphi_{2} & \phi_{2} \\
\vdots & \vdots & \vdots & \ddots & \vdots & \vdots \\
\lambda_{n}^{n-1} \varphi_{n} & \lambda_{n}^{n-2} \phi_{n} & \lambda_{n}^{n-3} \varphi_{n} & \cdots & \lambda_{n} \varphi_{n} & \phi_{n}
\end{array}\right|, \\
\Omega_{12}=\left|\begin{array}{cccccc}
\lambda_{1}^{n} \phi_{1} & \lambda_{1}^{n-2} \phi_{1} & \lambda_{1}^{n-3} \varphi_{1} & \cdots & \lambda_{1} \varphi_{1} & \phi_{1} \\
\lambda_{2}^{n} \phi_{2} & \lambda_{2}^{n-2} \phi_{2} & \lambda_{2}^{n-3} \varphi_{2} & \cdots & \lambda_{2} \varphi_{2} & \phi_{2} \\
\vdots & \vdots & \vdots & \ddots & \vdots & \vdots \\
\lambda_{n}^{n} \phi_{n} & \lambda_{n}^{n-2} \phi_{n} & \lambda_{n}^{n-3} \varphi_{n} & \cdots & \lambda_{n} \varphi_{n} & \phi_{n}
\end{array}\right|, \\
\Omega_{21}=\left|\begin{array}{cccccc}
\lambda_{1}^{n-1} \phi_{1} & \lambda_{1}^{n-2} \varphi_{1} & \lambda_{1}^{n-3} \phi_{1} & \cdots & \lambda_{1} \phi_{1} & \varphi_{1} \\
\lambda_{2}^{n-1} \phi_{2} & \lambda_{2}^{n-2} \varphi_{2} & \lambda_{2}^{n-3} \phi_{2} & \cdots & \lambda_{2} \phi_{2} & \varphi_{2} \\
\vdots & \vdots & \vdots & \ddots & \vdots & \vdots \\
\lambda_{n}^{n-1} \phi_{n} & \lambda_{n}^{n-2} \varphi_{n} & \lambda_{n}^{n-3} \phi_{n} & \cdots & \lambda_{n} \phi_{n} & \varphi_{n}
\end{array}\right|,
\end{gathered}
$$

2) for $n=2 k+1$,

$$
\begin{gathered}
\Omega_{11}=\left|\begin{array}{cccccc}
\lambda_{1}^{n-1} \varphi_{1} & \lambda_{1}^{n-2} \phi_{1} & \lambda_{1}^{n-3} \varphi_{1} & \cdots & \lambda_{1} \phi_{1} & \varphi_{1} \\
\lambda_{2}^{n-1} \varphi_{2} & \lambda_{2}^{n-2} \phi_{2} & \lambda_{2}^{n-3} \varphi_{2} & \cdots & \lambda_{2} \phi_{2} & \varphi_{2} \\
\vdots & \vdots & \vdots & \ddots & \vdots & \vdots \\
\lambda_{n}^{n-1} \varphi_{n} & \lambda_{n}^{n-2} \phi_{n} & \lambda_{n}^{n-3} \varphi_{n} & \cdots & \lambda_{n} \phi_{n} & \varphi_{n}
\end{array}\right|, \\
\Omega_{12}=\left|\begin{array}{cccccc}
\lambda_{1}^{n} \phi_{1} & \lambda_{1}^{n-2} \phi_{1} & \lambda_{1}^{n-3} \varphi_{1} & \cdots & \lambda_{1} \phi_{1} & \varphi_{1} \\
\lambda_{2}^{n} \phi_{2} & \lambda_{2}^{n-2} \phi_{2} & \lambda_{2}^{n-3} \varphi_{2} & \cdots & \lambda_{2} \phi_{2} & \varphi_{2} \\
\vdots & \vdots & \vdots & \ddots & \vdots & \vdots \\
\lambda_{n}^{n} \phi_{n} & \lambda_{n}^{n-2} \phi_{n} & \lambda_{n}^{n-3} \varphi_{n} & \cdots & \lambda_{n} \phi_{n} & \varphi_{n}
\end{array}\right|, \\
\Omega_{21}=\left|\begin{array}{cccccc}
\lambda_{1}^{n-1} \phi_{1} & \lambda_{1}^{n-2} \varphi_{1} & \lambda_{1}^{n-3} \phi_{1} & \cdots & \lambda_{1} \varphi_{1} & \phi_{1} \\
\lambda_{2}^{n-1} \phi_{2} & \lambda_{2}^{n-2} \varphi_{2} & \lambda_{2}^{n-3} \phi_{2} & \cdots & \lambda_{2} \varphi_{2} & \phi_{2} \\
\vdots & \vdots & \vdots & \ddots & \vdots & \vdots \\
\lambda_{n}^{n-1} \phi_{n} & \lambda_{n}^{n-2} \varphi_{n} & \lambda_{n}^{n-3} \phi_{n} & \cdots & \lambda_{n} \varphi_{n} & \phi_{n}
\end{array}\right|,
\end{gathered}
$$

Note that the eigenfunction $\psi_{k}=\left(\begin{array}{l}\phi_{k} \\ \varphi_{k}\end{array}\right)$ associated with the eigenvalue $\lambda_{k}$ has the following properties [29]

1) $\phi_{k}^{*}=\varphi_{k}, \lambda_{k}=-\lambda_{k}^{*}$;

2) $\phi_{k}^{*}=\varphi_{l}, \varphi_{k}^{*}=\phi_{l}, \lambda_{k}^{*}=-\lambda_{l}$, where $k \neq l$.

Based on the $N$-order solutions of the DNLS equation by determinant expression, we can get 


$$
q^{[2]}=\frac{\left(\lambda_{1} \varphi_{1} \phi_{2}-\lambda_{2} \phi_{1} \varphi_{2}\right)^{2}}{\left(-\lambda_{2} \phi_{2} \varphi_{1}+\lambda_{1} \varphi_{2} \phi_{1}\right)^{2}} q+2 i \frac{\left(\lambda_{1}^{2}-\lambda_{2}^{2}\right) \phi_{1} \phi_{2}\left(\lambda_{1} \varphi_{1} \phi_{2}-\lambda_{2} \phi_{1} \varphi_{2}\right)}{\left(-\lambda_{2} \phi_{2} \varphi_{1}+\lambda_{1} \varphi_{2} \phi_{1}\right)^{2}},
$$

with $\phi_{1}$ and $\varphi_{1}$ given by Equation (11).

Set $a$ and $c$ to be two real constants, substituting $q=c \exp \left(i\left(a x+\left(-c^{2}+a\right) a t\right)\right)$ into the spectral problem Equation (5) and Equation (6), the eigenfunction $\psi_{k}$ [13] associated with $\lambda_{k}$ is given by

$$
\begin{aligned}
& \left(\begin{array}{l}
\phi_{k}\left(x, t, \lambda_{k}\right) \\
\varphi_{k}\left(x, t, \lambda_{k}\right)
\end{array}\right) \\
& =\left(\begin{array}{l}
\varpi 1\left(x, t, \lambda_{k}\right)[1, k]+\varpi 2\left(x, t, \lambda_{k}\right)[1, k]+\varpi 1^{*}\left(x, t,-\lambda_{k}^{*}\right)[2, k]+\varpi 2^{*}\left(x, t,-\lambda_{k}^{*}\right)[2, k] \\
\varpi 1\left(x, t, \lambda_{k}\right)[2, k]+\varpi 2\left(x, t, \lambda_{k}\right)[2, k]+\varpi 1^{*}\left(x, t,-\lambda_{k}^{*}\right)[1, k]+\varpi 2^{*}\left(x, t,-\lambda_{k}^{*}\right)[1, k]
\end{array}\right) .
\end{aligned}
$$

Here

$$
\begin{aligned}
& \left(\begin{array}{l}
\varpi 1\left(x, t, \lambda_{k}\right)[1, k] \\
\varpi 1\left(x, t, \lambda_{k}\right)[2, k]
\end{array}\right) \\
& =\left(\begin{array}{c}
\exp \left(\frac{\sqrt{s}\left(x+2 \lambda_{k}^{2} t+\left(-c^{2}+a\right) t\right)}{2}+\frac{1}{2}\left(i\left(a x+\left(-c^{2}+a\right) a t\right)\right)\right) \\
\frac{i a-2 i \lambda_{k}^{2}+\sqrt{s}}{2 \lambda_{k} c} \exp \left(\frac{\sqrt{s}\left(x+2 \lambda_{k}^{2} t+\left(-c^{2}+a\right) t\right)}{2}-\frac{1}{2}\left(i\left(a x+\left(-c^{2}+a\right) a t\right)\right)\right)
\end{array}\right), \\
& \left(\begin{array}{l}
\varpi 2\left(x, t, \lambda_{k}\right)[1, k] \\
\varpi 2\left(x, t, \lambda_{k}\right)[2, k]
\end{array}\right) \\
& =\left(\begin{array}{c}
\exp \left(-\frac{\sqrt{s}\left(x+2 \lambda_{k}^{2} t+\left(-c^{2}+a\right) t\right)}{2}+\frac{1}{2}\left(i\left(a x+\left(-c^{2}+a\right) a t\right)\right)\right) \\
\frac{i a-2 i \lambda_{k}^{2}-\sqrt{s}}{2 \lambda_{k} c} \exp \left(-\frac{\sqrt{s}\left(x+2 \lambda_{k}^{2} t+\left(-c^{2}+a\right) t\right)}{2}-\frac{1}{2}\left(i\left(a x+\left(-c^{2}+a\right) a t\right)\right)\right)
\end{array}\right), \\
& s=-a^{2}-4 \lambda_{k}^{4}-4 \lambda_{k}^{2}\left(c^{2}-a\right) .
\end{aligned}
$$

1) Breather solution: Under the choice one paired eigenvalue $\lambda_{1}=\alpha_{1}+i \beta_{1}$ and $\lambda_{2}=-\alpha_{1}+i \beta_{1}$ and the eigenfunction $\psi_{k}$ associated with $\lambda_{k}$ from a periodic solution, then the breather solution has the following form as the Equation (53) from [13]. We give that the breather solutions gradually become into Peregrine rogue wave solutions under the condition $\frac{1}{2} c^{2}<a$ in Figure 2.

2) Two soliton-like solutions: Under the choice $\lambda_{1}=i \beta_{1}, \lambda_{2}=i \beta_{2}$ and the eigenfunction $\psi_{k}$ associated with $\lambda_{k}$ from a periodic solution, then

$$
\left|q_{s p}\right|^{2}=c^{2}+\frac{4\left(\beta_{1}^{2}-\beta_{2}^{2}\right)^{2}}{\left(\beta_{2} \frac{g_{1}^{*}}{g_{1}}-\beta_{1} \frac{g_{2}^{*}}{g_{2}}\right)\left(\beta_{2} \frac{g_{1}}{g_{1}^{*}}-\beta_{1} \frac{g_{2}}{g_{2}^{*}}\right)}-\operatorname{Re}\left(\frac{4 c\left(\beta_{1}^{2}-\beta_{2}^{2}\right)}{\beta_{1} \frac{g_{1}^{*}}{g_{1}}-\beta_{2} \frac{g_{2}^{*}}{g_{2}}}\right),
$$




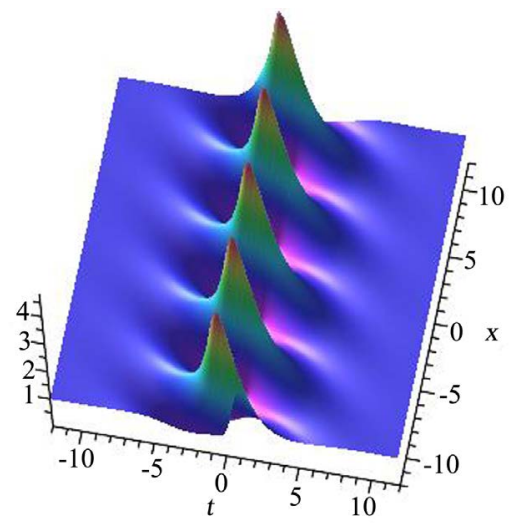

(a)

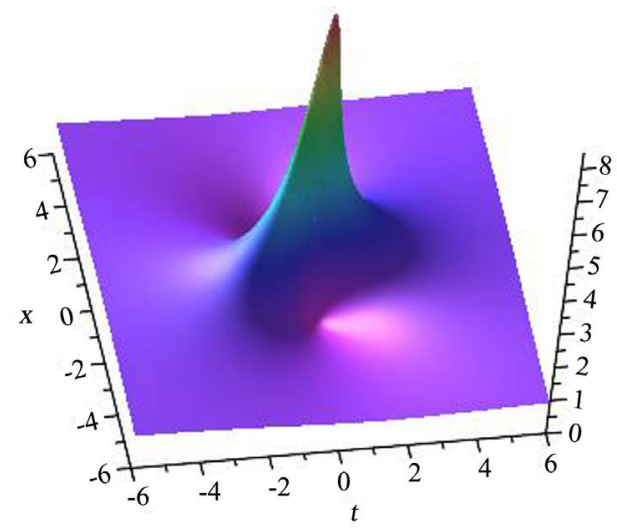

(b)

Figure 2. (Color online) The density plot of the breather solution $\left|q_{b s}\right|^{2}$ with the parameters $a=1, c=1, \alpha_{1}=-\frac{1}{2}, \beta_{1}=-\frac{1}{2}+\hat{m}$, (a) $\hat{m}=\frac{1}{5}$, (b) $\hat{m}=\frac{1}{50}$. When $\hat{m} \rightarrow 0$, the breather solution $\left|q_{b s}\right|^{2}$ gradually becomes a Peregrine rogue wave solution (i.e. Figure 1(a)).

$$
\begin{gathered}
g_{i}=2\left(1+\frac{2 \beta_{i}^{2}+a}{2 c \beta_{i}}\right) \cosh \left(\frac{1}{2} \sqrt{h_{i}}\left(x-2 \beta_{i}^{2} t+\left(-c^{2}+a\right) t\right)\right) \\
+i \frac{\sqrt{h_{i}}}{c \beta_{i}} \sinh \left(\frac{1}{2} \sqrt{h_{i}}\left(x-2 \beta_{i}^{2} t+\left(-c^{2}+a\right) t\right)\right), \\
h_{i}=4 \beta_{i}^{2} c^{2}-\left(2 \beta_{i}^{2}+a\right)^{2}, i=1,2 .
\end{gathered}
$$

In the limitation $\beta_{1} \rightarrow \frac{1}{2}\left(\sqrt{c^{2}-2 a}-c\right)$ and $\beta_{2} \rightarrow \frac{1}{2}\left(-\sqrt{c^{2}-2 a}-c\right)$, $\left(\lambda_{c_{1}}=\frac{1}{2}\left(\sqrt{c^{2}-2 a}-c\right) i, \quad \lambda_{c_{2}}=\frac{1}{2}\left(-\sqrt{c^{2}-2 a}-c\right) i\right.$, the values of spectral parameters are the zero point of $h_{i}$ and give the soliton-like solutions with different polarities), the two soliton-like solutions $\left|q_{s p}\right|^{2}$ gradually degenerate into the Peregrine rogue waves (see in Figure 3) under the condition $\frac{3}{8} c^{2}<a<\frac{1}{2} c^{2}$.

Note that a single soliton-like solution is given in the Equation (49) from [13]. From the expression of a single soliton-like solution, we can get the bright and dark soliton and phase solution directly. The expressions of the two solitons are complex, so the complex relations are obtained: two solitons, two phase solutions and soliton-phase solutions.

3) A special example: Based on the above two mechanisms, we found that $\frac{1}{2} c^{2}=a$ is boundary points. In order to better understand this state, we consider the equivalent solution in this case (when $\frac{1}{2} c^{2}=a$, the seed solution has the following form: $q=c \exp \left(i\left(\frac{1}{2} c^{2} x-\frac{1}{4} c^{4} t\right)\right)$, which can be given by (13) with $n=1)$.

$$
q_{m s}=2 i \frac{\Omega_{11} \Omega_{12}}{\Omega_{11}^{* 2}}
$$




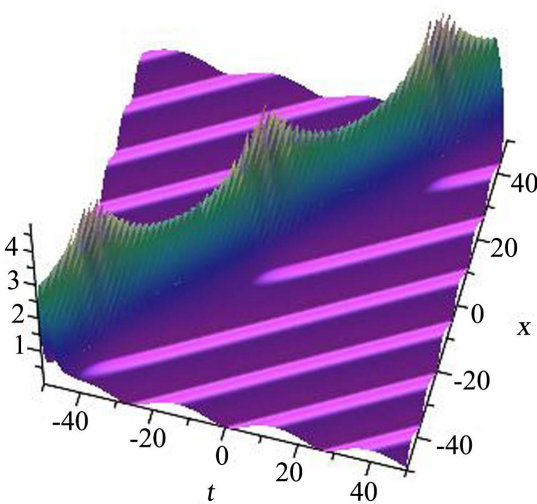

(a)

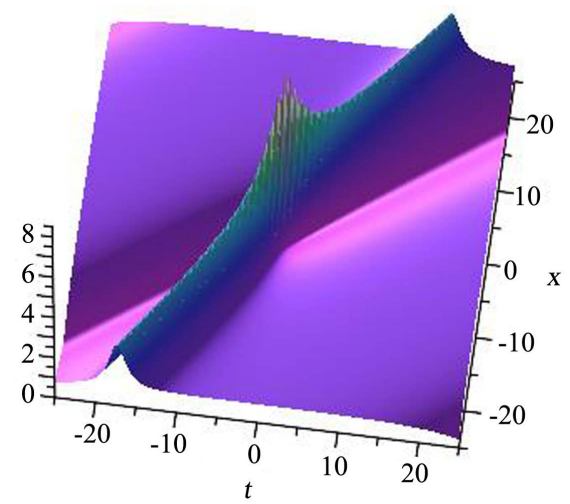

(b)

Figure 3. (Color online) The density plot of the two soliton-like solution $\left|q_{s p}\right|^{2}$ with the parameters $a=\frac{7}{16}, c=1, \quad \beta_{1}=-\frac{1}{2}-\frac{1}{8} \sqrt{2}+\hat{m}, \quad \beta_{2}=-\frac{1}{2}+\frac{1}{8} \sqrt{2}+\hat{m}, \quad$ (a) $\hat{m}=\frac{1}{5}$, (b) $\hat{m}=\frac{1}{50}$. When $\hat{m} \rightarrow 0$, the two soliton-like solution $\left|q_{b s}\right|^{2}$ gradually becomes a Peregrine rogue wave solution (i.e. Figure $1(\mathrm{c})$ ).

$$
\begin{gathered}
\Omega_{11}=\left|\begin{array}{lll}
\lambda_{1}^{2} \varphi_{1} & \lambda_{1} \phi_{1} & \varphi_{1} \\
\lambda_{2}^{2} \varphi_{2} & \lambda_{2} \phi_{2} & \varphi_{2} \\
\lambda_{3}^{2} \varphi_{3} & \lambda_{3} \phi_{3} & \varphi_{3}
\end{array}\right|, \\
\Omega_{12}=\left|\begin{array}{lll}
\lambda_{1}^{3} \phi_{1} & \lambda_{1} \phi_{1} & \varphi_{1} \\
\lambda_{2}^{3} \phi_{2} & \lambda_{2} \phi_{2} & \varphi_{2} \\
\lambda_{3}^{3} \phi_{3} & \lambda_{3} \phi_{3} & \varphi_{3}
\end{array}\right|, \\
\phi_{1}=\exp \left[i\left(\lambda_{1}^{2} x+2 \lambda_{1}^{4} t\right)\right], \varphi_{1}=\exp \left[-i\left(\lambda_{1}^{2} x+2 \lambda_{1}^{4} t\right)\right], \lambda_{1}=i h, \\
\phi_{2}=\exp \left[i\left(\lambda_{2}^{2} x+2 \lambda_{2}^{4} t\right)\right], \varphi_{2}=\exp \left[-i\left(\lambda_{2}^{2} x+2 \lambda_{2}^{4} t\right)\right], \lambda_{2}=i l+s_{k}, \\
\phi_{3}=\exp \left[i\left(\lambda_{3}^{2} x+2 \lambda_{3}^{4} t\right)\right], \varphi_{3}=\exp \left[-i\left(\lambda_{3}^{2} x+2 \lambda_{3}^{4} t\right)\right], \\
\lambda_{3}=i l-s_{k}, s_{k} \in R \cup i R .
\end{gathered}
$$

A special Peregrine rogue generated by a breather solution and phase solution (see Figure 4), which is given by the trivial seed (zero solution).

Based on the above analysis, we can get the relations between breather solutions, phase solutions, soliton solutions and rogue waves. The condition $a>\frac{3}{8} c^{2}$ on (c, a)-plane in Figure 5 can give the generation of the Peregrine rogue waves, so we can generate the Peregrine rogue waves from the degeneration of three kinds of solutions, namely, the breather solutions, the soliton-like solutions and the phase solutions.

\section{Summary}

In the paper, we provide the formation mechanism of Peregrine rogue waves of the DNLS equation, which is used to describe the propagation of Alfven waves in plasma physics and sub-picosecond or femtosecond pulses in nonlinear optics. 


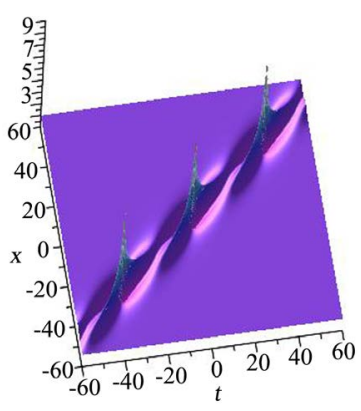

(a)

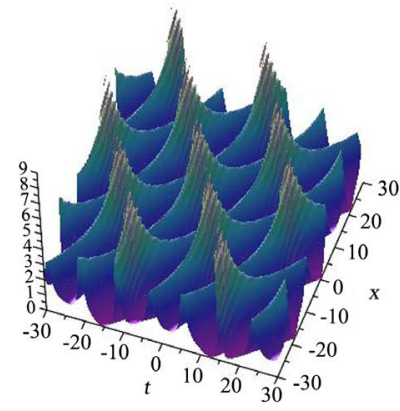

(c)

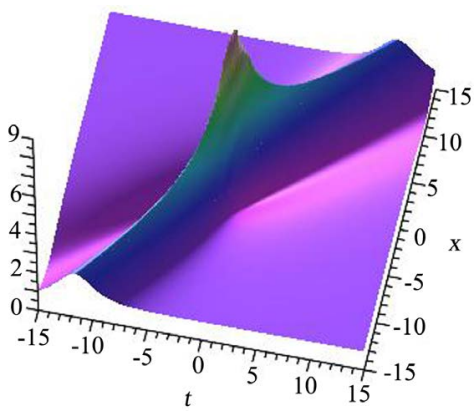

(b)

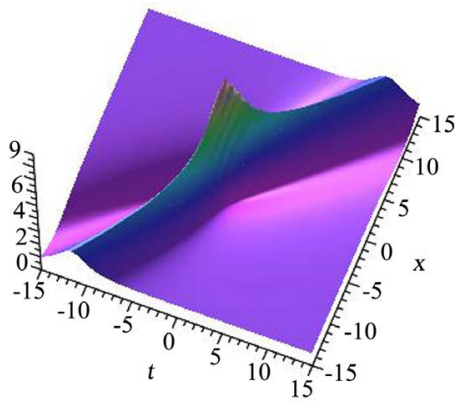

(d)

Figure 4. (Color online) The density plot of the mixed solution $\left|q_{m s}\right|^{2}$ (the solution includes breather solution and phase solution.) with the parameters $l=-\frac{1}{2}, h=-\frac{1}{2}$, (a) $s_{1}=\frac{1}{5}$, (b) $s_{1}=\frac{1}{50}$, (c) $s_{1}=\frac{1}{5} i$, (d) $s_{1}=\frac{1}{50} i$. When $\hat{m} \rightarrow 0$, the mixed solution $\left|q_{m s}\right|^{2}$ gradually becomes a Peregrine rogue wave solution (i.e. Figure 1(b)).

Relations between breather solutions, phase solutions, soliton solutions and rogue waves

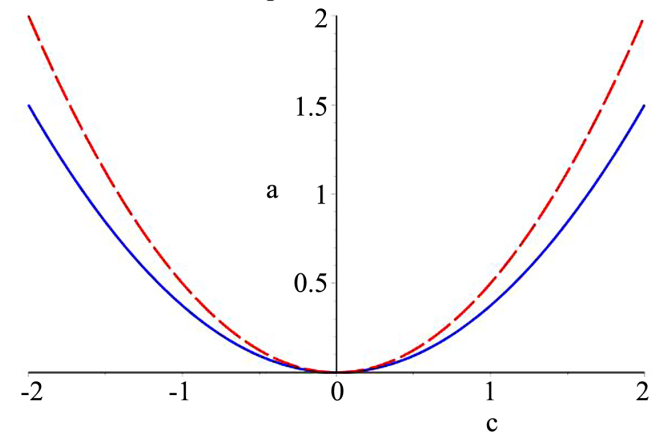

Figure 5. (Color online) Under the condition $a-\frac{1}{2} c^{2}>0$ on ((c), (a))-plane, the Peregrine rogue waves can be given by the degeneration of breather solutions, but under the condition $a-\frac{1}{2} c^{2}<0$ on ((c), (a))-plane, the Peregrine rogue waves can be given by the degeneration of soliton-like solutions (their related structures of soliton and phase solutions). Note that under the condition $a-\frac{1}{2} c^{2}=0$ on ((c), (a))-plane, the Peregrine rogue waves can be given by the degeneration of the breather (phase) solutions. The curve (red, long dash) is $a-\frac{1}{2} c^{2}=0$, but the curve (blue, solid) is $a-\frac{3}{8} c^{2}=0$. 
The bound state of two soliton-like solutions is figuratively illustrated in Figure 2 and Figure 3. In one case, the breather solution is the bound state periodically oscillates. In another case, the bound state of two soliton-like solutions has the collision of two solitons. Collisions of two soliton-like structures can cause such exciting rogue wave phenomenon. These results are helpful to the realization and observation of physical experiments.

\section{Acknowledgements}

This work is supported by the National Natural Science Foundation of China under Grant No. 11601187, Natural Science Foundation of Ningbo under Grant No. 2018A610197 and Major SRT Project of Jiaxing University.

\section{Conflicts of Interest}

The authors declare no conflicts of interest regarding the publication of this paper.

\section{References}

[1] Kharif, C., Pelinovsky, E. and Slunyaev, A. (2009) Rogue Waves in the Ocean. Springer, Berlin.

[2] Solli, D.R., Ropers, C., Koonath, P. and Jalali, B. (2007) Optical Rogue Waves. Nature, 450, 1054-1057. https://doi.org/10.1038/nature06402

[3] Kibler, B., Fatome, J., Finot, C., Millot, G., Dias, F., Genty, G., Akhmediev, N. and Dudley, J.M. (2010) The Peregrine Soliton in Nonlinear Fibre Optics. Nature Physics, 6, 790-795. https://doi.org/10.1038/nphys1740

[4] Chabchoub, A., Hoffmann, N.P. and Akhmediev, N. (2011) Rogue Wave Observation in a Water Wave Tank. Physical Review Letters, 106, Article ID: 204502. https://doi.org/10.1103/PhysRevLett.106.204502

[5] Chabchoub, A., Hoffmann, N., Onorato, M. and Akhmediev, N. (2012) Super Rogue Waves: Observation of a Higher-Order Breather in Water Waves. Physical Review $X$, 2, Article ID: 011015. https://doi.org/10.1103/PhysRevX.2.011015

[6] Zakharov, V.E. and Ostrovsky, L.A. (2009) Modulation Instability: The Beginning. Physica D: Nonlinear Phenomena, 238, 540-548. https://doi.org/10.1016/j.physd.2008.12.002

[7] Zakharov, V.E. and Gelash, A.A. (2013) Nonlinear Stage of Modulation Instability. Physical Review Letters, 111, Article ID: 054101. https://doi.org/10.1103/PhysRevLett.111.054101

[8] Peregrine, D.H. (1983) Water Waves, Nonlinear Schrödinger Equations and Their Solutions. Journal of the Australian Mathematical Society Series B Applied Mathematics, 25, 16-43. https://doi.org/10.1017/S0334270000003891

[9] Akhmediev, N., Ankiewicz, A. and Taki, M. (2009) Waves That Appear from Nowhere and Disappear without a Trace. Physics Letters A, 373, 675-678. https://doi.org/10.1016/j.physleta.2008.12.036

[10] Kuznetsov, E.A. (1977) Solitons in a Parametrically Unstable Plasma. Soviet Physics-Doklady, 22, 507-508.

[11] Ma, Y.C. (1979) The Perturbed Plane-Wave Solutions of the Cubic Schrödinger Equation. Studies in Applied Mathematics, 60, 43-58. 
https://doi.org/10.1002/sapm197960143

[12] Akhmediev, N.N. and Korneev, V.I. (1987) Modulation Instability and Periodic Solutions of Nonlinear Schrödinger Equation. Theoretical and Mathematical Physics, 69, 1089-1093. https://doi.org/10.1007/BF01037866

[13] Xu, S.W., He, J.S. and Wang, L.H. (2011) The Darboux Transformation of the Derivative Nonlinear Schrödinger Equation. Journal of Physics A: Mathematical and Theoretical, 44, Article ID: 305203. https://doi.org/10.1088/1751-8113/44/30/305203

[14] Guo, B.L., Ling, L.M. and Liu, Q.P. (2012) High-Order Solutions and Generalized Darboux Transformations of Derivative Nonlinear Schrödinger Equations. Studies in Applied Mathematics, 130, 317-344. https://doi.org/10.1111/j.1467-9590.2012.00568.x

[15] Xu, S.W., Wang, L.H., Erdlyi, R. and He, J.S. (2019) Degeneracy in Bright-Dark Solitons of the Derivative Nonlinear Schrodinger Equation. Applied Mathematics Letters, 87, 64-72. https://doi.org/10.1016/j.aml.2018.07.021

[16] Xu, S.W., He, J.S. and Mihalache, D. (2019) Rogue Waves Generation through Multiphase Solutions Degeneration for the Derivative Nonlinear Schrödinger Equation. Nonlinear Dynamics, 97, 2443-2452. https://doi.org/10.1007/s11071-019-05140-0

[17] Wu, Z., Xu, S.W., Wu, T.W. and Zhou, H.Q. (2019) The Interaction and Degeneracy of Mixed Solutions for Derivative Nonlinear Schrödinger Equation. Journal of Applied Mathematics and Physics, 7, 2650-2657.

https://doi.org/10.4236/jamp.2019.711180

[18] Tzoar, N. and Jain, M. (1981) Self-Phase Modulation in Long-Geometry Optical Waveguide. Physical Review A, 23, 1266-1270. https://doi.org/10.1103/PhysRevA.23.1266

[19] Anderson, D. and Lisak, M. (1983) Nonlinear Asymmetric Self-Phase Modulation and Self-Steepening of Pulses in Long Optical Waveguides. Physical Review A, 27, 1393-1398. https://doi.org/10.1103/PhysRevA.27.1393

[20] Agrawal, G.P. (2001) Nonlinear Fibers Optics. 3rd Edition, Academic, New York.

[21] Mj $\phi$ lhus, E. (1976) On the Modulational Instability of Hydromagnetic Waves Parallel to the Magnetic field. Journal of Plasma Physics, 16, 321-334. https://doi.org/10.1017/S0022377800020249

[22] Spangler, S.P. (1997) Nonlinear Evolution of MHD Waves at The Earth's Bow Shock: Opinions on the Confrontation between Theory, Simulations, and Measurements. In: Hada, T. and Matsumoto, H., Eds., Nonlinear Waves and Chaos in Space Plasmas, Terra Scientific Publishing Company, Tokyo. 171-224.

[23] Kaup, D.J. and Newell, A.C. (1978) An Exact Solution for a Derivative Nonlinear Schrödinger Equation. Journal of Mathematical Physics, 19, 798-801. https://doi.org/10.1063/1.523737

[24] Huang, N.N. and Chen, Z.Y. (1990) Alfven Solitons. Journal of Physics A: Mathematical and General, 23, 439-453. https://doi.org/10.1088/0305-4470/23/4/014

[25] Ichikawa, Y.H. and Watanabe, S. (1977) Solitons and Envelope Solitons in Collissionless Plasmas. Journal de Physique Colloques, 38, C6.15-C6.26. https://doi.org/10.1051/jphyscol:1977603

[26] Kawata, T. and Inoue, H. (1978) Exact Solutions of the Derivative Nonlinear Schrödinger Equation under the Nonvanishing Conditions. Journal of the Physical Society of Japan, 44, 1968-1976. https://doi.org/10.1143/JPSJ.44.1968

[27] Kawata, T., Kobayashi, N. and Inoue, H. (1979) Soliton Solution of the Derivative 
Nolinear Schrödinger Equation. Journal of the Physical Society of Japan, 46, 1008-1015. https://doi.org/10.1143/JPSJ.46.1008

[28] Chen, X.J. and Lam, W.K. (2004) Inverse Scattering Transform for the Derivative Nonlinear Schrödinger Equation with Nonvanishing Boundary Conditions. Physical Review E, 69, Article ID: 066604. https://doi.org/10.1103/PhysRevE.69.066604

[29] Imai, K. (1999) Generalization of Kaup-Newell Inverse Scattering Formulation and Darboux Transformation. Journal of the Physical Society of Japan, 68, 355-359. https://doi.org/10.1143/JPSJ.68.355

[30] Steudel, H. (2003) The Hierarchy of Multi-Soliton Solutions of the Derivative Nonlinear Schrödinger Equation. Journal of Physics A: Mathematical and General, 36, 1931-1946. https://doi.org/10.1088/0305-4470/36/7/309

[31] Neugebauer, G. and Meinel, R. (1984) General N-Soliton Solution of the AKNS Class on Arbitrary Background. Physics Letters A, 100, 467-470. https://doi.org/10.1016/0375-9601(84)90827-2

[32] Matveev, V.B. and Salle, M.A. (1991) Darboux Transfromations and Solitons. Springer, Berlin.

[33] Ablowitz, M.J., Kaup, D.J., Newell, A.C. and Segur, H. (1973) Nonlinear Evolution Equations of Physical Significance. Physical Review Letters, 31, 125-127. https://doi.org/10.1103/PhysRevLett.31.125

[34] Ablowitz, M.J. and Clarkson, P.A. (1991) Solitons, Nonlinear Evolution Equations and Inverse Scattering. Cambridge University Press, Cambridge.

https://doi.org/10.1017/CBO9780511623998

[35] El Bertola, M.G.A. and Tovbis, A. (2016) Rogue Waves in Multiphase Solutions of the Focusing Nonlinear Schrödinger Equation. Proceedings of the Royal Society A, 472, Article ID: 20160340. https://doi.org/10.1098/rspa.2016.0340

[36] Slunyaev, A.V. and Pelinovsky, E.N. (2016) Role of Multiple Soliton Interactions in the Generation of Rogue Waves: The Modified Korteweg-de Vries Framework. Physical Review Letters, 117, Article ID: 214501. https://doi.org/10.1103/PhysRevLett.117.214501

[37] Herink, G., Kurtz, F., Jalali, B., Solli, D.R. and Ropers, C. (2017) Real-Time Spectral Interferometry Probes the Internal Dynamics of Femtosecond Soliton Molecules. Science, 356, 50-54. https://doi.org/10.1126/science.aal5326

[38] Liu, X.M., Yao, X.K. and Cui, Y.D. (2018) Real-Time Observation of the Buildup of Soliton Molecules. Physical Review Letters, 121, Article ID: 023905.

https://doi.org/10.1103/PhysRevLett.121.023905

[39] Melchert, O., Willms, S., Bose, S., Yulin, A., Roth, B., Mitschke, F., Morgner, U., Babushkin, I. and Demircan, A. (2019) Soliton Molecules with Two Frequencies. Physical Review Letters, 123, Article ID: 243905. https://doi.org/10.1103/PhysRevLett.123.243905

[40] Pelinovsky, E.N., Shurgalina, E.G., Sergeeva, A.V., Talipova, T.G., El, G.A. and Grimshaw, R.H.J. (2013) Two-Soliton Interaction as an Elementary Act of Soliton Turbulence in Integrable Systems. Physics Letters A, 377, 272-275. https://doi.org/10.1016/j.physleta.2012.11.037

[41] Xu, G., Gelash, A., Chabchoub, A., Zakharov, V. and Kibler, B. (2019) Breather Wave Molecules. Physical Review Letters, 122, Article ID: 084101. https://doi.org/10.1103/PhysRevLett.122.084101

[42] Lou, S.Y. (2020) Soliton Molecules and Asymmetric Solitons in Three Fifth Order Systems via Velocity Resonance. Journal of Physics Communications, 4, Article ID: 041002. https://doi.org/10.1088/2399-6528/ab833e 